\title{
A Link Between Scattering Resonances and Dilation Analytic Resonances in Few Body Quantum Mechanics
}

\author{
George A. Hagedorn ${ }^{\star}$ \\ The Rockefeller University, New York, New York 10021, USA
}

\begin{abstract}
We study the ( 2 cluster $) \rightarrow(2$ cluster $)$ scattering amplitudes for classes of two, three, and four particle dilation analytic Schrödinger operators whose two-body potentials fall off exponentially. As functions of the energy, these amplitudes are shown to have meromorphic continuations on certain Riemann surfaces. We prove that all poles of these continuations are necessarily bound states or dilation analytic resonances [i.e., eigenvalues of $H(\theta)$ for some $\theta$ ].
\end{abstract}

\section{Introduction}

Within the theory of dilation analytic Schrödinger operators there are two very different definitions of resonances. A dilation analytic resonance is a complex eigenvalue of an operator $H(\theta)$. A scattering resonance is a pole of the analytic continuation of a scattering amplitude. Scattering resonances are directly related to quantities measured in experiments; dilation analytic resonances are easier to estimate by numerical methods.

In this paper a connection between the two definitions is established. We study classes of two, three, and four particle dilation analytic Schrödinger operators, whose two body potentials fall off exponentially (Yukawa potentials are allowed). On certain Riemann surfaces, we construct the meromorphic continuations of the ( 2 cluster) $\rightarrow$ ( 2 cluster) scattering amplitudes. Our main result (Theorem 3.1) is that within the meromorphy domains which we obtain, every scattering resonance is a dilation analytic resonance.

In the literature, previous results of this genre $[2,9,12]$ deal only with the two body case. Various subtleties of the different definitions of resonances are discussed in [14] and in the references given there.

The paper is organized as follows: In Sect. 2, we establish notation, state hypotheses on potentials, prove some preliminary lemmas, and recall the (2 cluster $) \rightarrow(2$ cluster) $T$-matrix formula of [8]. Our main result (Theorem 3.1$)$ is

* Supported in part by the National Science Foundation under Grant PHY 78-08066 
proved in Sect. 3 by combining dilation analyticity with the $T$-matrix formula and the two body ideas of $[5,6,12]$.

\section{Preliminaries}

In this section we establish notation, state hypotheses on potentials, slightly alter the resolvent formulas of [7], and recall the (2 cluster) $\rightarrow(2$ cluster $) T$-matrix formula of [8].

\section{A. Notation}

The Schrödinger operator for $N$ particles in $m$ dimensions is

$$
\tilde{H}=-\sum_{i=1}\left(2 m_{i}\right)^{-1} \Delta_{i}+\sum_{i<j} V_{i j}\left(r_{i}-r_{j}\right) \quad \text { on } \quad L^{2}\left(\mathbb{R}^{N m}\right) \text {. }
$$

We remove the center of mass motion from $\tilde{H}$ to obtain $H=H_{0}+\sum_{i<j} V_{i j}$ on $\mathscr{H}=L^{2}\left(\mathbb{R}^{(N-1) m}\right)$.

A cluster decomposition $D=\left\{C_{i}\right\}_{i=1}^{k}$ is a partition of $\{1, \ldots, N\}$ into $k$ disjoint clusters $C_{i}$. We define $H_{D}=H_{0}+V_{D}$, where $V_{D}$ is the sum of all $V_{i j}$ with $i$ and $j$ in the same cluster of $D$. $\mathscr{H}$ may be decomposed as

$$
\mathscr{H}_{1} \otimes \mathscr{H}_{2} \otimes \ldots \otimes \mathscr{H}_{k} \otimes \mathscr{H}(D)
$$

so that

$$
H_{D}=h_{1} \otimes 1 \otimes \ldots \otimes 1+1 \otimes h_{2} \otimes \ldots \otimes 1+\ldots+1 \otimes \ldots \otimes h_{k} \otimes 1+1 \otimes \ldots \otimes 1 \otimes K_{D} .
$$

The Hamiltonian $h_{i}$ corresponds to the energy of the particles in cluster $C_{i}$ alone. $K_{D}$ is the kinetic energy of the centers of mass of the clusters of $D$.

For each $i$, we choose eigenfunctions $\eta_{j}^{(i)}$ of $h_{i}$ so that $\left\{\eta_{j}^{(i)}\right\}$ is an orthonormal basis for the subspace of $\mathscr{H}_{i}$ spanned by the eigenfunctions of $h_{i}$.

A channel $\alpha$ is a cluster decomposition $D(\alpha)$ together with an eigenfunction $\eta^{(i)} \in\left\{\eta_{j}^{(i)}\right\}$ for each $h_{i}$. The threshold corresponding to $\alpha$ is $E_{\alpha}=\sum_{i=1}^{k} E_{i}$, where $h_{i} \eta^{(i)}$ $=E_{i} \eta^{(i)}$. We define $\psi_{\alpha}=\eta^{(1)} \otimes \eta^{(2)} \otimes \ldots \otimes \eta^{(k)}$, and let $P_{\alpha}: \mathscr{H} \rightarrow \mathscr{H}$ denote the orthogonal projection onto all vectors of the form $\psi_{\alpha} \otimes \phi$, where $\phi \in \mathscr{H}(D(\alpha))$ is arbitrary. We identify the range of $P_{\alpha}$ with $\mathscr{H}_{\alpha}=\mathscr{H}(D(\alpha))$ by identifying $\psi_{\alpha} \otimes \phi$ with $\phi$. We let $P_{D}$ be the sum of all $P_{\alpha}$ with $D(\alpha)=D$.

When $\alpha$ is a 2 cluster channel we let $M_{\alpha}$ denote the reduced mass associated with the coordinate $\zeta$ between the centers of mass of the clusters of $D(\alpha)$. We also define $\phi_{\alpha}^{0}(X, k)=e^{i k \zeta} \psi_{\alpha}\left(x_{\alpha}\right)$, where $X=\left(x_{\alpha}, \zeta\right)$.

We refer the reader to $[3,10,11]$ for definitions and theorems concerning objects related to dilation analyticity $\left(H(\theta), \sigma_{\text {ess }}(H(\theta))\right.$, thresholds, etc.).

\section{B. Hypotheses on Potentials}

Throughout this paper we assume the following hypotheses:

(i) $H=H_{0}+\sum_{i<j} \lambda_{i j} V_{i j}$ is an $N$ particle Schrödinger operator on $\mathscr{H}=L^{2}\left(\mathbb{R}^{(N-1) m}\right)$, where $N \leqq 4$ and $m \geqq 3$. 
(ii) Each $V_{i j}$ may be factored as $V_{i j}=U_{i j} W_{i j}$ so that $U_{i j}$ and $W_{i j}$ are dilation analytic in $S_{\theta_{0}}=\left\{\theta:|\operatorname{Im} \theta| \leqq \theta_{0}\right\}$, where $\theta_{0}<\pi / 2$.

(iii) There exists $\gamma_{0}>0$ such that $e^{\gamma_{0} e^{\theta}\left|x_{i j}\right|} U_{i j}\left(\theta, x_{i j}\right)$ and $e^{\gamma_{0} e^{\theta}\left|x_{i j}\right|} W_{i j}\left(\theta, x_{i j}\right)$ belong to $L^{p}\left(\mathbb{R}^{m}, d x_{i j}\right)$ for some $p>m$ and all $\theta \in S_{\theta_{0}}$.

(iv) Bound state energies of three body subsystems are non-positive when $N=4$.

Remark. Balslev [1] and Simon [13] have given sufficient conditions for hypothesis (iv) to hold (see also [7, Theorem II.10]). Yukawa potentials satisfy all of the above conditions (i)-(iv).

\section{Modification of Resolvent Formulas}

In time independent scattering theory, scattering information is obtained from a detailed analysis of the resolvent of the Hamiltonian near the continuous spectrum. The resolvent is singular near the spectrum, and it is useful to obtain resolvent formulas which isolate the singularities.

We will use the 2, 3, and 4 particle resolvent formulas of [7], which have the form:

$$
(z-H)^{-1}=\sum_{D}\left(z-H_{D}\right)^{-1} P_{D} \sum_{\ell=1}^{L(D)} F_{\ell, D} Z_{\ell, D}(z) .
$$

In these formulas, the singular part has been isolated in the factor $\left(z-H_{D}\right)^{-1}$ in the sense that $Z_{\ell, D}(z)$ is fairly well behaved. More precisely, for each $\phi$ in a dense subspace of $\mathscr{H}, Z_{\ell, D}(z) \phi \in \mathscr{H}$ has continuous boundary values as $z$ approaches the continuous spectrum except on a closed set of measure zero. The $F_{\ell, D}$ factor is multiplication by a function which falls off in some intercluster coordinate. The fall off provides smoothness in momentum space, and this smoothness is used to make sense of the restriction of the Fourier transform of $P_{D} F_{\ell, D} Z_{\ell, D}(x \pm i 0) \phi$ to the surface which corresponds to energy $x$. All of the scattering information is contained in these restricted Fourier Transforms (see $[7,8]$ ).

For our purposes, we apply the standard dilation analytic techniques $[3,11]$ to Eq. (2.1) to obtain :

$$
(z-H(\theta))^{-1}=\sum_{D}\left(z-H_{D}(\theta)\right)^{-1} P_{D}(\theta) \sum_{\ell=1}^{L(D)} F_{\ell, D}(\theta) Z_{\ell, D}(\theta, z) .
$$

By using the explicit formulas of Sect. IV of [7], we see that if $D$ has exactly two clusters, then

$$
Z_{\ell, D}(\theta, z)=B_{\ell, D}(\theta, z) C(\theta, z)+B_{\ell, D}(\theta, z)(1-M(\theta, z))^{-1} M(\theta, z) C(\theta, z) .
$$

If we choose $z$ to be far from the spectrum of $H(\theta)$, then the inverse term in Eq. (2.3) can be expanded by Geometric series. By resumming this series and analytically continuing in $z$, we obtain the following formula when $D$ has exactly two clusters:

$$
F_{\ell, D}(\theta) Z_{\ell, D}(\theta, z)=P_{D}(\theta)\left(1+\left[V(\theta)-V_{D}(\theta)\right](z-H(\theta))^{-1}\right) .
$$

The properties of $F_{\ell, D}(\theta)$ and $Z_{\ell, D}(\theta, z)$ which we require are summarized in Lemma 2.1. We note that Eqs. (2.1), (2.2), and (2.3) depend on the choice of certain functions $\varrho_{i j}, \varrho_{i j k}, \varrho_{i j, k \ell}$, etc. The available choices depend on the potentials, and our choice differs from those of [7] and [8]. Our $\varrho$ functions are given by the 
following definition which depends on a number $\gamma>0$. The value of $\gamma$ is fixed in Lemma 2.1.

Definition. Let $\xi$ be the coordinate from the center of mass of particles $i$ and $j$ to particle $k$. Let $\zeta$ be the coordinate from the center of mass of particles $i, j$, and $k$ to particle $\ell$. Let $\eta$ be the coordinate from the center of mass of particles $i$ and $j$ to the center of mass of particles $k$ and $\ell$. We define

$$
\left.\begin{array}{r}
\varrho_{i j, k}(\xi)=e^{-\gamma|\xi|} \\
\varrho_{i j k}(\zeta)=e^{-\gamma|\zeta|} \\
\varrho_{i j, k \ell}(\eta)=e^{-\gamma|\eta|}
\end{array}\right\} .
$$

Lemma 2.1. Assume hypotheses (i)-(iv). The 2, 3, and 4 body resolvent formulas of [7] (Eqs. (IV.1), (IV.6), and (IV.10) of [7], respectively) may be written in the form of Eq. (2.2). Fix $D$ with exactly two clusters, and represent $Z_{\ell, D}$ by Eq. (2.3). For each set of generic couplings $\left\{\lambda_{i j}\right\}$, there exists $\gamma>0$ so that if the $\varrho$ functions are chosen by Eq. (2.5), then the following hold:

(a) Each $F_{\ell, D}$ is a @ function which falls off exponentially in the coordinate between the clusters of $D$.

(b) For each $\ell \leqq L(D), C(\bar{\theta}, \bar{z})^{*} B_{\ell, D}(\bar{\theta}, \bar{z})^{*} F_{\ell, D}(\bar{\theta}) *=P_{D}(\theta)(z \notin \sigma(H(\theta)))$.

(c) For each $\ell \leqq L(D)$ and each pair $i j$,

$$
X(\theta, z)=W_{i j}(\theta) C(\bar{\theta}, \bar{z})^{*} M(\bar{\theta}, \bar{z})^{*}\left[1-M(\bar{\theta}, \bar{z})^{*}\right]^{-1} B_{\ell, D}(\bar{\theta}, \bar{z})^{*}: \mathscr{H} \rightarrow \mathscr{H}
$$

is an analytic operator valued function for $z \notin \sigma(H(\theta))$.

(d) Let $X(\theta, z)$ be as in (c). If $\operatorname{Im} \theta=0$ and $\delta>1$, then, as an operator from $\mathscr{H}$ to $L_{-\delta}^{2}\left(\mathbb{R}^{(N-1) m}\right)=\left\{f:\left(1+x^{2}\right)^{-\delta / 2} f(x) \in L^{2}\left(\mathbb{R}^{(N-1) m}\right)\right\}, X(\theta, z)$ has continuous extensions to $\sigma_{\text {ess }}(H)$ from above and below in the complement of a closed set $\mathscr{E}$ of measure zero.

Proof. Parts (a) and (b) follow trivially from the explicit formulas of [7].

To prove (c) and (d) for some $\gamma>0$, we must modify all the estimates of Sect. V of [7] to control the growth of the functions $\varrho^{-1}$. However, a careful inspection of the proofs of those estimates reveals the following fact: If Lemma V.4 of [7] holds for our $\varrho$ functions with $\gamma>0$, then all of the required estimates hold for that value of $\gamma$.

Thus, it suffices to prove Lemma V.4 of [7] for our $\varrho$ functions with some $\gamma>0$. We will show $\varrho_{i j, k}^{-1}(\theta) P_{i j}(\theta) U_{i k}(\theta)$ is bounded on $L^{2}\left(\mathbb{R}^{(N-1) m}\right)$ whenever $|\operatorname{Im} \theta|<\theta_{0}$. The other operators of Lemma V.4 of [7] can be treated in the same fashion. Fix $\theta$ with $|\operatorname{Im} \theta|<\theta_{0}$. Then

$$
\begin{aligned}
\varrho_{i j, k}^{-1}(\theta) P_{i j}(\theta) U_{i k}(\theta)= & P_{i j}(\theta) U_{i k}(\theta) \varrho_{i j, k}^{-1}(\theta) \\
= & {\left[P_{i j}(\theta) e^{a\left|x_{i j}\right|}\left(1-\Delta_{i j}\right)^{1 / 2}\right] } \\
& \cdot\left[\left(1-\Delta_{i j}\right)^{-1 / 2} U_{i k}(\theta) e^{\gamma_{0}\left|e^{\theta} x_{i k}\right|}\right] \\
& \cdot\left[e^{-\gamma_{0}\left|e^{\theta} x_{i k}\right|} e^{-a\left|x_{i j}\right|} \varrho_{i j, k}^{-1}(\theta, \xi)\right] .
\end{aligned}
$$

Theorem II.9 of [7] bounds the first factor for some value of $a>0$ which depends continuously on $\theta$. The second factor is controlled by the same method used to control the analogous factor in the proof of Lemma V.4 of [7]. The third factor is a product of exponentials. Since $\xi=C_{1} x_{i j}+C_{2} x_{i k}$, the triangle inequality shows that 
the product is bounded whenever $\gamma<\gamma_{1}$. Here $\gamma_{1}$ depends continuously on $a$, and hence $\theta$.

Since $\theta_{0}<\pi / 2$, it is easy to see that $\gamma_{1}$ can be bounded away from 0 when $|\operatorname{Im} \theta| \leqq \theta_{0}$. This implies the existence of a $\gamma$ for which the lemma holds.

Remarks. 1. For $N=2,3, \mathscr{E}$ is the set of eigenvalues of $H$. For $N=4$ it may also contain spurious zeros (see [7]).

2. For all but a discrete set of $(\operatorname{Im} \theta)^{\prime} \mathrm{s},(d)$ can be improved to include boundary values of $X(\theta, z)$ on $\sigma_{\text {ess }}(H(\theta)) \backslash \mathscr{E}(\theta)(\mathscr{E}(\theta)$ is discrete for each allowed $\theta)$. The bad values of $\operatorname{Im} \theta$ are those for which new resonance thresholds are appearing.

\section{The (2 Cluster $) \rightarrow(2$ Cluster $)$ T-Matrix}

Proposition 2.2. Assume hypotheses (i)-(iv). Assume $\left\{\lambda_{i j}=1\right\}$ is a set of generic couplings. Let $\alpha$ and $\beta$ both be 2 cluster channels. Let $S_{\alpha \beta}$ denote the block of the $S$-matrix for scattering from channel $\beta$ to channel $\alpha$. Then, for a dense set off's in $\mathscr{H}_{\alpha}$ and a dense set of $g$ 's in $\mathscr{H}_{\beta}$,

$$
\begin{aligned}
& \left\langle f, S_{\alpha \beta} g\right\rangle-\delta_{\alpha \beta}\langle f, g\rangle \\
& \quad=-2 \pi i \int_{\mathbb{R}^{2 m}} \overline{\hat{f}(k)} \hat{g}\left(k^{\prime}\right) \delta\left(k^{2} / 2 M_{\alpha}+E_{\alpha}-k^{\prime 2} / 2 M_{\beta}-E_{\beta}\right) T_{\alpha \beta}\left(k, k^{\prime}\right) d k d k^{\prime},
\end{aligned}
$$

where

$$
\begin{aligned}
& T_{\alpha \beta}\left(k, k^{\prime}\right)=(2 \pi)^{-m} \int_{\mathbb{R}^{(N-1) m}} \overline{\phi_{\alpha}^{0}(X, k)}\left[V(X)-V_{D(\alpha)}(X)\right] \\
& \quad \cdot \sum_{l=1}^{L(D(\beta))}\left[\left(Z_{\ell, D(\beta)}\left(k^{\prime 2} / 2 M_{\beta}+E_{\beta}-i 0\right)\right)^{*} F_{\ell, D(\beta)}^{*} \phi_{\beta}^{0}\left(\cdot, k^{\prime}\right)\right](X) d X .
\end{aligned}
$$

Moreover, $T_{\alpha \beta}\left(k, k^{\prime}\right)$ is continuous in $k$ and $k^{\prime}$ whenever $k^{\prime 2} / 2 M_{\beta}+E_{\beta} \notin \mathscr{E}$.

Proof. This follows from Theorem 1.2 of [8] where Eq. (2.6) contains the $\varrho$ functions chosen in [8]. Lemma 2.1 is all that is required to modify the proof of Theorem 1.2 of [8] to accomodate the $\varrho$ functions which we chose in Eq. (2.5).

Remark. When $N=2$, formulas (2.4) and (2.6) give the familiar formula:

$$
T\left(k, k^{\prime}\right)=(2 \pi)^{-m} \int e^{-i k x} V(x)\left(e^{i k^{\prime} x}+\left[\left(k^{\prime 2} / 2 M+i 0-H\right)^{-1} V e^{i k^{\prime}}\right](x)\right) d x .
$$

\section{Meromophic Continuation of the $(2 \rightarrow 2)$ Scattering Amplitude}

In this section we prove our main result (Theorem 3.1). We begin by choosing the amplitude to be continued. We then describe the surface on which the continuation is defined.

Given any two 2 cluster channels $\alpha$ and $\beta$, the associated $T$-matrix, $T_{\alpha \beta}\left(\mathbf{k}, \mathbf{k}^{\prime}\right)$, depends on the outgoing and incoming momenta $\mathbf{k} \in \mathbb{R}^{m}$ and $\mathbf{k}^{\prime} \in \mathbb{R}^{m}$ (The dimension of space is $m \geqq 3$ ). We pick unit vectors $\hat{e}_{1}$ and $\hat{e}_{2}$ in $\mathbb{R}^{m}$ and restrict attention to $\mathbf{k}=k \hat{e}_{1}$ and $\mathbf{k}^{\prime}=k^{\prime} \hat{e}_{2}$. Furthermore, due to energy conservation, the physically interesting values of $k$ and $k^{\prime}$ satisfy $k^{2} / 2 M_{\alpha}+E_{\alpha}=k^{\prime 2} / 2 M_{B}+E_{B}=E$. So, 
we define $f_{\alpha \beta}(E)=T_{\alpha \beta}\left(k \hat{e}_{1}, k^{\prime} \hat{e}_{2}\right)$, where $k$ and $k^{\prime}$ are determined by

$$
\left.\begin{array}{rl}
k & =\left[2 M_{\alpha}\left(E-E_{\alpha}\right)\right]^{1 / 2} \\
k^{\prime} & =\left[2 M_{\beta}\left(E-E_{\beta}\right)\right]^{1 / 2}
\end{array}\right\} .
$$

The square roots are chosen so that $k$ and $k^{\prime}$ are both positive when the energy $E$ is in the physical region $E>\operatorname{Max}\left\{E_{\alpha}, E_{\beta}\right\}$. For simplicity we concentrate on $f_{\alpha \beta}(E)$ rather than $T_{\alpha \beta}\left(\mathbf{k}, \mathbf{k}^{\prime}\right)$ (see Corollary 3.2 for more information concerning $T_{\alpha \beta}$ ).

We will construct the meromorphic continuation of $f_{\alpha \beta}(E)$ on a Riemann surface $\mathscr{F}_{\alpha \beta}$, which is an open subset of the Riemann surface $\mathscr{F}$ usually associated $[3,11]$ with the dilation analytic Schrödinger operator $H$. Every threshold is a branch point of $\mathscr{F}$. We identify each function $g$ on $\mathscr{F}$ with a function $g(\theta, z)$, where $z \in \mathbb{C} \backslash \sigma_{\text {ess }}(H(\theta))$ and $g(\theta, z)$ is locally independent of $\theta$ for fixed $z \notin \sigma_{\text {ess }}(H(\theta))$. By an abuse of notation we represent $\mathscr{F}$ as $\bigcup_{\theta}\left\{z \in \mathbb{C}: z \notin \sigma_{\text {ess }}(H(\theta))\right\}$.

Theorem 3.1. Assume hypotheses (i)-(iv) for $H=H_{0}+\sum_{i<j} \lambda_{i j} V_{i j}$, and let $\alpha$ and $\beta$ be any two 2 cluster channels for $H$.

Then, for each set of generic couplings $\left\{\hat{\lambda}_{i j}\right\}$, there exist $\sigma>0$ and a closed set $\mathscr{E} \subseteq \mathbb{R}$ of measure zero so that the following holds: The amplitude $f_{\alpha \beta}(E)$ has a meromorphic continuation from $\mathscr{F}_{\text {Physical }}=\left\{E+i 0 \in \mathbb{R} \backslash \mathscr{E}: E>\operatorname{Max}\left\{E_{\alpha}, E_{\beta}\right\}\right\}$ to the connected component $\mathscr{F}_{\alpha \beta}$ of

$$
\mathscr{F}_{\alpha \beta}^{0}=\bigcup_{\theta \in S_{\theta_{0}}}\left\{E: E \in \mathbb{C} \backslash \sigma_{\text {ess }}(H(\theta)),\left|\operatorname{Im}\left(k e^{\theta}\right)\right|<\sigma,\left|\operatorname{Im}\left(k^{\prime} e^{\theta}\right)\right|<\sigma\right\} \cong \mathscr{F}
$$

which contains $\mathscr{F}_{\text {Physical }}\left[\right.$ Eqs. (3.1) determine $\left.k, k^{\prime}\right]$. All poles of $f_{\alpha \beta}$ in $\mathscr{F}_{\alpha \beta}$ are bound states or dilation analytic resonances for $H$.

Remarks. 1. Yukawa potentials satisfy all the hypotheses of Theorem 3.1.

2. The generic couplings are precisely those for which no cluster Hamiltonian has a threshold resonance or threshold bound state. This set of couplings is large in the sense that its complement is a closed set of measure zero [7, Sect. VI].

3. The region $\mathscr{F}_{\alpha \beta}^{0}$ is represented in the conclusion of the theorem as a union of sets $\mathscr{G}(\theta) \subseteq \mathbb{C} \backslash \sigma_{\text {ess }}(H(\theta))$. $\mathscr{G}(\theta)$ is the intersection of three regions. The first region is $\mathbb{C} \backslash \sigma_{\text {ess }}(H(\theta))$. The second is the set of all points $E$ which lie in the "inside" region bounded by the parabola $\left|\operatorname{Im}\left(e^{\theta}\left[2 M_{\alpha}\left(E-E_{\alpha}\right)\right]^{1 / 2}\right)\right|=\sigma$. This region contains $E_{\alpha}$ and is symmetric about the "cut" $\left\{E_{\alpha}+\mu e^{-2 \theta}: \mu \in[0, \infty)\right\} \subseteq \sigma_{\text {ess }}(H(\theta))$. The third region is a similar "solid parabola" containing $E_{\beta}$ and which is symmetric about $\left\{E_{\beta}+\mu e^{-2 \theta}: \mu \in[0, \infty)\right\} \subseteq \sigma_{\text {ess }}(H(\theta))$.

4. For suitable $\alpha$ and $\beta, \mathscr{F}_{\alpha \beta}$ is large in some sense. For example, let $N=3$ and choose $\alpha=\beta$, where $E_{\alpha}$ is the lowest 2 cluster threshold. Choose $\theta_{1}$ satisfying $0<\theta_{1}<\theta_{0}$ so that $|\operatorname{Im} \theta|<\theta_{1}$ implies that every threshold $E_{\delta}$ satisfies $\left|\operatorname{Im}\left(e^{\theta}\left[2 M_{\alpha}\left(E_{\delta}-E_{\alpha}\right)\right]^{1 / 2}\right)\right|<\sigma$.

Then $\mathscr{F}_{\alpha \alpha}$ contains all points $z$ on the "unphysical sheets" of $\mathscr{F}$ which lie over $\left\{z:-2 \theta_{1}<\arg \left(z-E_{\alpha}\right)<0\right\}$. All dilation analytic resonances which are eigenvalues of the operators $H(\theta)$ with $|\operatorname{Im} \theta|<\theta_{1}$ must lie in $\mathscr{F}_{\alpha \alpha}$.

5. In Theorem 3.1, hypothesis (iii) may be weakened. Exponential fall off of $U_{i j}(\theta)$ is required, but $W_{i j}(\theta)$ need only lie in $L_{\delta}^{p}\left(\mathbb{R}^{m}\right)+L_{\delta}^{\infty}\left(\mathbb{R}^{m}\right)$ for some $p>m$ and $\delta>m / 2$. We have used Hypothesis (iii) to avoid rewriting a few of the estimates of 
Sect. V of [7] in the proof of Lemma 2.1. By the weakening of hypotheses we can obtain a larger set $\mathscr{F}_{\alpha \beta}$.

Proof of Theorem 3.1. If $\theta$ is real, than Eq. (2.3) and (2.6) show:

$$
f_{\alpha \beta}(E)=\sum_{\sim D(\alpha)} \sum_{\ell=1}^{L(D(\beta))}\left\langle\Psi_{i j}(\bar{\theta}, \bar{E}), \Lambda_{\ell, i j}(\theta, E+i 0) \Phi_{\ell}(\theta, E)\right\rangle,
$$

where

$$
\begin{aligned}
\Psi_{i j}(\bar{\theta}, \bar{E}) & =\left(U_{i j}(\theta)\right)^{*} \phi_{\alpha}^{0}(\bar{k}, \bar{\theta}) \\
\Phi_{\ell}(\theta, E) & =\left(F_{\ell, D(\beta)}(\bar{\theta})\right)^{*} \phi_{\beta}^{0}\left(k^{\prime}, \theta\right) \\
\Lambda_{\ell, i j}(\theta, E) & =W_{i j}(\theta)\left(Z_{\ell, D(\beta)}(\bar{\theta}, \overline{E+i 0)})^{*}\right.
\end{aligned}
$$

and $\sum_{\sim D(\alpha)}$ indicates summation over all pairs $i j$ with $i$ and $j$ in different clusters of $D(\alpha)$. As usual $E$ determines $k$ and $k^{\prime}$ by Eq. (3.1).

Since the couplings are generic, $\psi_{\alpha}(\theta)$ and $\psi_{\beta}(\theta)$ have exponential fall off for $\theta \in S_{\theta_{0}}\left(\right.$ Note $\left.\theta_{0}<\pi / 2\right)$. Thus, Lemma 2.1 (a) shows that

$$
\Phi_{\ell}(\theta, E, X)=\left(F_{\ell, D(\beta)}(\bar{\theta})\right)^{*}(\zeta) e^{i e^{\theta} k^{\prime} \hat{e}_{2} \cdot \zeta} \psi_{\beta}\left(\theta, x_{\beta}\right)
$$

is an analytic $\mathscr{H}$-valued function of $E$ for fixed $\theta \in S_{\theta_{0}}$, as long as $\left|\operatorname{Im} e^{\theta} k^{\prime}\right|<\sigma_{1}$. The rate of falloff of $F_{\ell, D(\beta)}$ (which is a $\varrho$ function) determines $\sigma_{1}$.

Using a similar argument, we see that $\Psi_{i j}(\bar{\theta}, \bar{E})$ is a conjugate analytic $L_{\delta}^{2}$-valued function of $E$ for each fixed $\theta \in S_{\theta_{0}}$, whenever, $\left|\operatorname{Im} e^{\theta} k\right|<\sigma_{2}$. This argument uses hypothesis (iii) and the representation of $x_{i j}$ as a linear combination of clustered Jacobi coordinates for $D(\beta)$.

For each $\theta \in S_{\theta_{0}}$, Lemma 2.1 (c) shows that $\Lambda_{\ell, i j}(E, \theta): \mathscr{H} \rightarrow L_{-\delta}^{2}$ is meromorphic for $E \notin \sigma_{\text {ess }}(H(\theta))$.

We can now construct the continuation of $f_{\alpha \beta}$. Let $\sigma=\operatorname{Min}\left\{\sigma_{1}, \sigma_{2}\right\}$, and represent $f_{\alpha \beta}(E)$ by Eq. (3.2). By Lemma 2.1 and the observations above, this representation is valid for $E \in \widetilde{F}_{\text {Physical }}$. Furthermore, the right hand side of Eq. (3.2) is meromorphic in $E$ and independent of $\theta$ whenever $E$ belongs to

$$
\mathscr{G}(\theta)=\left\{E \in \mathbb{C} \backslash \sigma_{\mathrm{ess}}(H(\theta)):\left|\operatorname{Im} e^{\theta} k\right|<\sigma,\left|\operatorname{Im} e^{\theta} k^{\prime}\right|<\sigma\right\} .
$$

The usual dilation analytic tricks $[3,11]$ show that $f_{\alpha \beta}$ is $\theta$-independent. Thus, by alternately changing $E$ and $\theta$, we see that $f_{\alpha \beta}(E)$ is meromorphic in $\mathscr{F}_{\alpha \beta}$.

Poles of $f_{\alpha \beta}$ in $\mathscr{F}_{\alpha \beta}$ can only arise from poles of $\Lambda_{\ell, i j}(\theta, E)$. Poles of $\Lambda_{\ell, i j}(\theta, E)$ can arise only from poles of $\left(1-M(\bar{\theta}, \bar{E})^{*}\right)^{-1}$ in Eq. (2.3), where $E \in \mathbb{C} \backslash \sigma_{\text {ess }}(H(\theta))$. For $N=2$ and $N=3$, these poles must be bound states or dilation analytic resonances (see Proposition V.1 and V.2 of [7]). For $N=4$ these poles can be bound states, dilation analytic resonances, or "spurious zeros".

Let $E$ be a spurious zero. To complete the proof, we must show $C(\bar{\theta}, \bar{z})^{*}\left(1-M(\bar{\theta}, \bar{z})^{*}\right)^{-1} M(\bar{\theta}, \bar{z})^{*}$ has no pole at $z=E$. This is equivalent to showing $\mathscr{C}(\bar{\theta}, \bar{z})^{*}\left(1-\mathscr{M}(\bar{\theta}, \bar{z})^{*}\right)^{-1} \mathscr{M}(\bar{\theta}, \bar{z})^{*}$ has no pole at $z=E$, where we let $\mathscr{A}, \mathscr{M}$, and $\mathscr{C}$ be the operators of Eq. IV.4 of [7]. Using the explicit formula for $\mathscr{M}(z, \theta)$ and the resolvent formula $\left(z-H_{D}(\theta)\right)^{-1}=\left(z-H_{0}(\theta)\right)^{-1}+\left(z-H_{D}(\theta)\right)^{-1} V_{D}(\theta)\left(z-H_{0}(\theta)\right)^{-1}$, it is easy to see that every pole of $\mathscr{C}(\bar{\theta}, \bar{z})^{*}\left(1-\mathscr{M}(\bar{\theta}, \bar{z})^{*}\right)^{-1} \mathscr{M}(\bar{\theta}, \bar{z})^{*}$ gives rise to a pole of $\mathscr{C}(\bar{\theta}, \bar{z})^{*}\left(1-\mathscr{M}(\bar{\theta}, \bar{z})^{*}\right)^{-1} \mathscr{A}(\bar{\theta}, \bar{z})^{*}$. Since $E$ is spurious, this last operator valued function has no pole at $E$.

Thus, spurious zeros do not give rise to poles of $f_{\alpha \beta}$. 
Remarks 1. Another proof that spurious zeros do not give poles of $f_{\alpha \beta}$ can be obtained by replacing Eq. (3.2) with Eqs. (2.4) and (2.6).

2. Suppose $E_{0} \in \mathscr{F}_{\alpha \beta}$ is a pole of $f_{\alpha \beta}(E)$. Assume $E_{0}$ is a simple eigenvalue of $H(\theta)$ with eigenfunction $\psi(\theta)$. By replacing Eq. (3.2) with Eq. (2.4) and (2.6), we can compute the residue of the pole of $f_{\alpha \beta}(E)$ at $E_{0}$. The result is

$$
\begin{gathered}
(2 \pi)^{-m}\left(\int \overline{\phi_{\alpha}^{0}(X, \bar{\theta}, \bar{k})}\left[V(\bar{\theta}, X)^{*}-V_{D(\alpha)}(\bar{\theta}, X)^{*}\right] \psi(\theta, X) d X\right) \\
.\left(\int \overline{\psi(\bar{\theta}, Y)}\left[V(\theta, Y)-V_{D(\beta)}(\theta, Y)\right] \phi_{\beta}^{0}\left(Y, \theta, k^{\prime}\right) d Y\right) .
\end{gathered}
$$

Corollary 3.2. Assume the hypotheses of Theorem 3.1. Assume the couplings are generic, and let $T_{\alpha \beta}(E): L^{2}\left(S^{m-1}\right) \rightarrow L^{2}\left(S^{m-1}\right)$ be the "on-shell" T-matrix for scattering from channel $\beta$ to channel $\alpha$. Then $T_{\alpha \beta}(E)$ is a meromorphic Hilbert-Schmidt operator valued function for $E \in \mathscr{F}_{\alpha \beta}$. Its poles must be dilation analytic resonances.

Proof. The integral kernel of $T_{\alpha \beta}(E)$ is $f_{\alpha \beta}\left(E, \hat{e}_{1}, \hat{e}_{2}\right)$. Theorem 3.1 establishes the meromorphic behaviour of $f_{\alpha \beta}\left(E, \hat{e}_{1}, \hat{e}_{2}\right)$, and it is clear that

$$
\int_{S^{m-1}} \int_{S^{m-1}}\left|f_{\alpha \beta}\left(E, \hat{e}_{1}, \hat{e}_{2}\right)\right|^{2} d \hat{e}_{1} d \hat{e}_{2}
$$

is finite. This implies the corollary.

Acknowledgements. It is a pleasure to thank Barry Simon and Percy Deift for their encouragement and useful discussions.

\section{References}

1. Balslev, E.: Absence of positive eigenvalues for Schrödinger operators. Arch. Rat. Mech. Anal. 59, 343-357 (1975)

2. Balslev, E.: Analytic scattering theory for two-body Schrödinger operators. J. Funct. Anal. 29, 375-396 (1978)

3. Balslev, E., Combes, J.M.: Spectral properties of many-body Schrödinger operators with dilatation-analytic interactions. Commun. math. Phys. 22, 280-294 (1971)

4. Combes, J.M., Thomas, L.E.: Asymptotic behavior of eigenfunctions of multiparticle Schrödinger operators. Commun. math. Phys. 34, 251-270 (1973)

5. Grossman, A., Wu, T.T.: Schrödinger scattering amplitude. I. J. Math. Phys. 2, 710-713 (1961)

6. Grossman, A., Wu, T.T. : Schrödinger scattering amplitude. III. J. Math. Phys. 3, 684-689 (1962)

7. Hagedorn, G.A. : Asymptotic completeness for classes of two, three, and four particle Schrödinger operators. Thesis, Princeton University (to appear)

8. Hagedorn, G. A. : Born series for ( 2 cluster $) \rightarrow(2$ cluster $)$ scattering of two, three, and four particle Schrödinger operators. Commun. math. Phys. (to appear) (1979)

9. Jensen, A. : Local distortion technique, resonances, and poles of the $S$-matrix. J. Math. Anal. Appl. 59, 505-513 (1977)

10. Reed, M., Simon, B.: Methods of modern mathematical physics, Vol. IV. Analysis of operators. New York, London: Academic Press 1978

11. Simon, B.: Quadratic form techniques and the Balslev-Combes theorem. Commun. math. Phys. 27, 1-10 (1972)

12. Simon, B.: Resonances in $n$-body quantum systems with dilatation analytic potentials and the foundations of time-dependent perturbation theory. Ann. Math. 97, 247-274 (1973)

13. Simon, B. : Absence of positive eigenvalues in a class of multiparticle quantum systems. Math. Ann. 207, 133-138 (1974)

14. Simon, B.: Resonances and complex scaling: A rigorous overview. Int. J. Q. Chem. (to appear)

Communicated by J. Glimm

Received November 28, 1978 